\title{
Performance of Turbo Product Code in Wimax
}

\author{
Trushita Chaware \\ Department of Information Technology \\ Thakur College of Engineering and Technology \\ Kandivali(E), Mumbai, India
}

\author{
Nileema Pathak \\ Computer Engineering Department \\ Atharva College of Engineering \\ Malad(W), Mumbai, India
}

\begin{abstract}
IEEE 802.16 is a standard for Broadband Wireless Access (BWA) air interface. 802.16e supports mobile broadband wireless access, which is an additional feature over its predecessors, which support fixed wireless access. Binary Convolutional Turbo Coding (CTC) is used as mandatory Forward Error Correction method in 802.16e. In this paper the performance of a simple and efficient optional coding scheme namely Turbo Product Code (TPC) is proposed for 802.16e system and is compared with CTC.
\end{abstract}

KEYWORDS-CTC; eBCH; AWGN; Code Rate; OFDM

\section{INTRODUCTION}

IEEE 802.16 standard system or commonly called Worldwide Interoperability for Microwave access (WiMAX), provides specifications for both fixed Line of Sight (LOS) communication in the range of $10-66 \mathrm{GHz}(802.16 \mathrm{c})$, and fixed, portable, Non-LOS communication in the range of 2$11 \mathrm{GHz}$ (802.16a, 802.16d). The IEEE 802.16e provides mobility and also enhanced performance. The architecture is based on scalable sub channel bandwidth using variable size FFT according to channel bandwidth. Forward Error correction is done by mandatory Convolution Codes. While doing channel coding, the limit on data rate is given by Shannon's limit is as $\mathrm{R}<\mathrm{W} \log _{2}(1+\mathrm{S} / \mathrm{N})$ bits/sec, which sets a limit on bandwidth and signal to noise ratio. Efficient communication systems are systems that permit a high rate of information to be communicated with the lowest possible power and least BER.

IEEE 802.16 wireless broadband standard is one such promising future wireless system, primarily because it offers the potential for high spectral efficiency, flexible spectrum options (2-6 GHz), scalable carrier bandwidth options (1.25 $\mathrm{MHz}$ to $20 \mathrm{MHz}$ ), multiple duplexing options (time and frequency division duplex), various sub channelization options, and also mobility.

\section{II. $\quad 802.16 \mathrm{SYSTEM}$}

IEEE 802.16 standard for Broadband Wireless Access and its associated industry consortium, WiMAX forum promises to offer high data rates over large areas to a large number of users where broadband is unavailable.

Taking the advantage of OFDM technique the physical layer of Wimax system is able to provide robust broadband service. The basic principle of OFDM is to divide a high-rate data stream into $\mathrm{N}$ lower rate streams and to transmit them at the same time over a number of subcarriers. In OFDM high bit rate data is divided into $\mathrm{N}$ low bit rate parallel data streams and then transmitted simultaneously with deferent frequencies. OFDM systems are implemented using a combination of fast Fourier Transform (FFT) and inverse fast Fourier Transform (IFFT) blocks. The effect of ISI on an OFDM signal can be further improved by the addition of a guard period to the start of each symbol. This guard period is a cyclic copy that extends the length of the symbol waveform [1]. The block diagram of a general Wimax system is shown in figure 1.

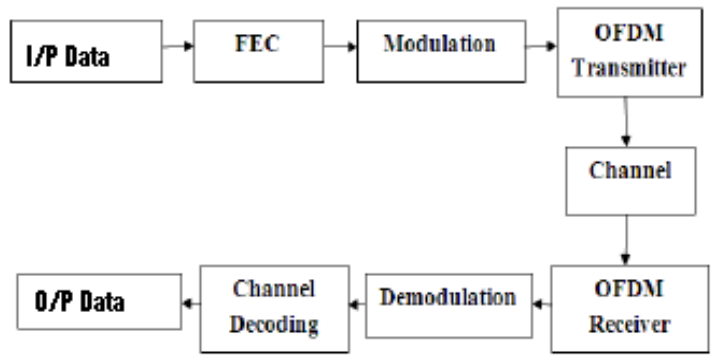

Fig.1. WiMAX System

The performance of this system can be further enhanced by using forward error correcting techniques. Commonly used error correcting method for a wireless medium for 802.16 standard is Convolutional Turbo Codes (CTC). Turbo Product Code (TPC) can be used instead of CTC and the performance of the wireless communication system can be analysed. TPC with $\mathrm{eBCH}$ as a constituent code provides further benefits, because $\mathrm{eBCH}$ code can be decoded easily using the syndrome method and it can be used for multiple random error correction. General points of comparison for CTC and TPC are that CTC perform best for low code rate applications while TPC perform best for high code rate applications. CTC will have difficulty achieving high data rates, whereas TPC can operate at high data rates. CTC exhibit error floor at BER below $10^{-5}$, while TPC error floor is less pronounced at lower BER values [2].

\section{CONVOLUTIONAL TURBO CODE}

In the Wireless MAN system, the Convolutional Codes $(\mathrm{CC})$ is the only mandatory coding scheme, all the others like CTC and TPC are optional. Convolutional codes map information to code bits sequentially by convolving a sequence of information bits with "generator" sequences. A convolutional encoder encodes $K$ information bits to $N>K$ code bits at one time step. Maximum Likelihood decoding can be done using Viterbi algorithm, other decoding algorithms 
such as SOVA (Soft Ouput Viterbi Algorithm) and BCJR ( Bahl, Cocke, Jelinek and Raviv) algorithm, can be used [3].

The CTC is a parallel concatenated convolutional code or turbo code. An overview of the CTC encoder is depicted in figure. 2. It consists of a CTC encoder with a natural rate of $1 / 3$ followed by an additional interleaver and a final puncturing to obtain the desired rate. The CTC encoder consists of two identical constituent encoders separated by a CTC internal interleaver. The constituent encoder has a natural rate of 2/4 and its minimal realization is depicted in the lower part of figure. 2. It consists of $\mathrm{m}=3$ memory elements and consumes two input bits per time instance and produces four output bits. Furthermore, the encoder is recursive and systematic. The CTC encoding procedure is described below. The two information bits A and B are fed directly to the output and in a first encoding step additionally into the constituent encoder, producing the parity bits Y1 and W1. Afterwards, in a second encoding step, the interleaved information symbols (A and B) are again fed into the constituent encoder, now producing the parity bits $\mathrm{Y} 2$ and $\mathrm{W} 2$. This means the info/code tuple of the CTC encoder is AB/ABY1W1Y2W2 and its natural rate is therefore $1 / 3$. CTC is decoded using the well known iterative decoding process using Log Maximum A Posteriori (MAP) algorithm, where the two decoders exchange information based on log likelihood ratio of the information bits. The complexity of this decoding process increases exponentially as they get closer to optimality.

\section{TURBO PRODUCT CODE}

A two-dimensional product code is built from two component codes with parameters $\mathrm{C} 1(\mathrm{n} 1, \mathrm{k} 1, \mathrm{~d} 1)$ and $\mathrm{C} 2$ (n 2, k2, d2), where ni, ki, di stands for code word length, number of information bits, and minimum hamming distance respectively [3]. The product code $\mathrm{P}=\mathrm{c} 1 \mathrm{x} \mathrm{c} 2$ is obtained by placing $(\mathrm{k} 1 \times \mathrm{k} 2)$ information bits in an array of $\mathrm{k} 1$ rows and $\mathrm{k} 2$ columns. The parameters of product code $\mathrm{P}$ are $\mathrm{n}=\mathrm{n} 1 \mathrm{X} \mathrm{n} 2$, $\mathrm{k}=\mathrm{k} 1 \mathrm{X} \mathrm{k} 2, \mathrm{~d}=\mathrm{d} 1 \mathrm{X} \mathrm{d} 2$ and code rate is $\mathrm{R}=\mathrm{R} 1 \mathrm{XR} 2$, where $\mathrm{Ri}$ is the code rate of $\mathrm{Ci}$.

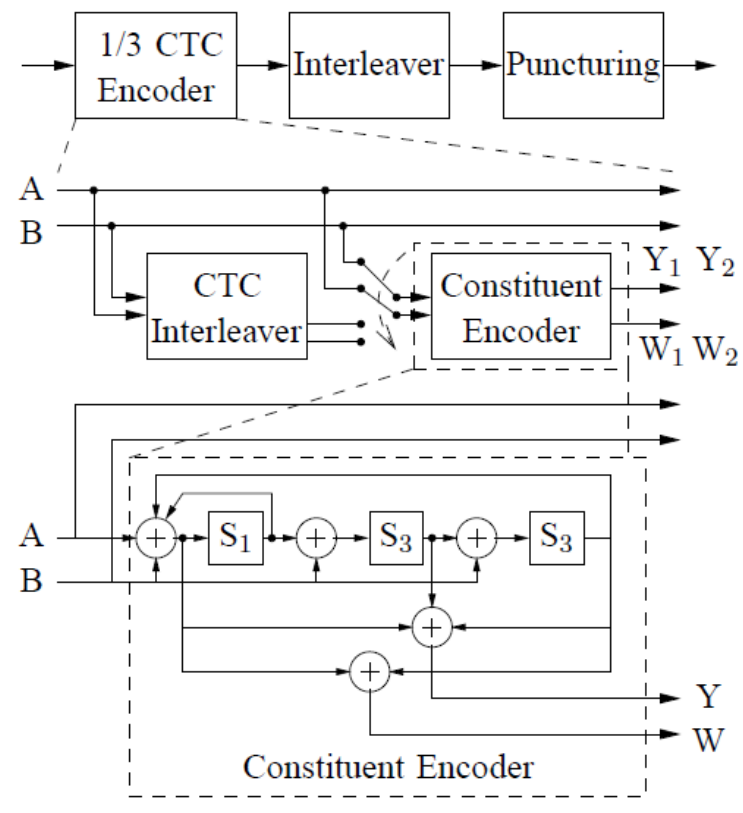

Fig.2. Convolutional Turbo Code Encoder

Thus very long block codes can be built with large minimum Hamming distance. Figure. 2 shows the procedure for construction of a 2D product code using two block codes $\mathrm{C} 1$ and $\mathrm{C} 2$. All the rows of matrix $\mathrm{P}$ are the code words of $\mathrm{C} 1$ and all the columns of matrix P are code words of C2 [4].

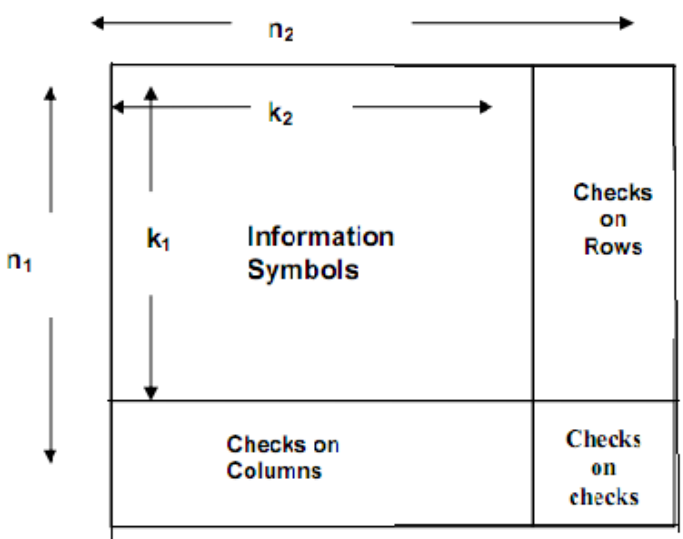

Fig.3. An example of a 2D product code constructed using two component codes

Other benefits in using TPC are as follows

- Longer battery life - Less transmit power required for the battery operated device using TPC.

- IP free encoder - No IP license required for encoding.

- Availability of IC's - Standard IC's are available providing the required functionality thus, the TPC implementation price increase is minimal.

- Lowest cost solution - A very low cost encoder, smaller batteries, smaller packages, it all adds up to reduced cost and improved performance [5].

\section{A. DECODING OF TPC}

\section{a) Soft Decoding of Linear Block Codes}

In hard decision decoding, received signal is sampled and the resulting voltages are compared with a single threshold. If a voltage is greater than the threshold it is considered to be definitely a 'one', regardless of how close it is to the threshold. If it is less, it is definitely 'zero'. In soft decision decoding we get not only the 1 or 0 decision but also an indication of how certain we are that the decision is correct. Few bits may be used as 'confidence' bits to indicate the certainty of soft decision. Turbo Product codes can be decoded by sequentially decoding the rows and columns of product code $\mathrm{P}$, in order to reduce decoding complexity. However, to achieve optimum performance, one must use Maximum Likelihood Decoding (soft decoding) of the component codes. Thus, we need softinput/ soft-output decoders to maintain optimum performance when decoding the rows and columns of product code $\mathrm{P}$.

\section{b) Chase Algorithm}

The Trellis based Maximum A posteriori Probability (MAP) decoding (soft decoding), used for CTC decoding, 
provides a good BER performance but it is very complex and computationally difficult.

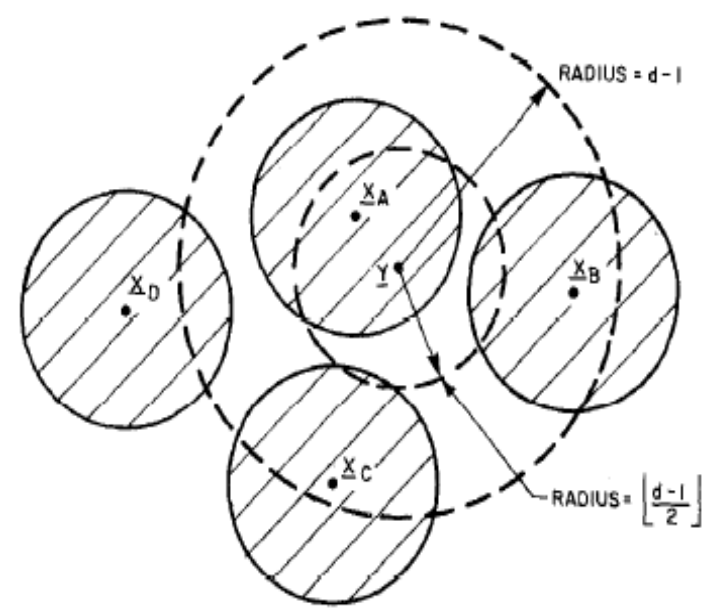

Fig.4. Geometric sketch for decoding with channel measurement information

Instead of using trellis based MAP algorithm, the Chase algorithm is repeatedly applied along rows and columns of TPC in order to obtain extrinsic information for each bit position. Chase algorithm is used to obtain soft output. Chase initially used a method of finding the Euclidean distance between code words $\left(2^{\mathrm{k}}\right)$ for filtering them for decoding. Each codeword is surrounded by a sphere of radius $(\mathrm{d}-1) / 2$. Thus, an unique codeword, or equivalently a unique error pattern, is obtained by a binary decoder if the received sequence is within one of these spheres. In our case there is a unique error pattern $\mathrm{Z}=\mathrm{Y} \mathrm{XOR} \mathrm{X}_{\mathrm{A}}$ within the sphere of radius $(\mathrm{d}-1) / 2$ which surrounds $\mathrm{Y}$ as shown in figure 4 [6].

\section{c) Chase - Pyndiah Algorithm}

This algorithm generates Test Patterns using least reliable bits $\mathrm{p}$. These least reliable bits are found using a reliability sequence obtained by soft decoding of received signal. Test patterns for $\mathrm{p}=2$ are shown in figure 5 .
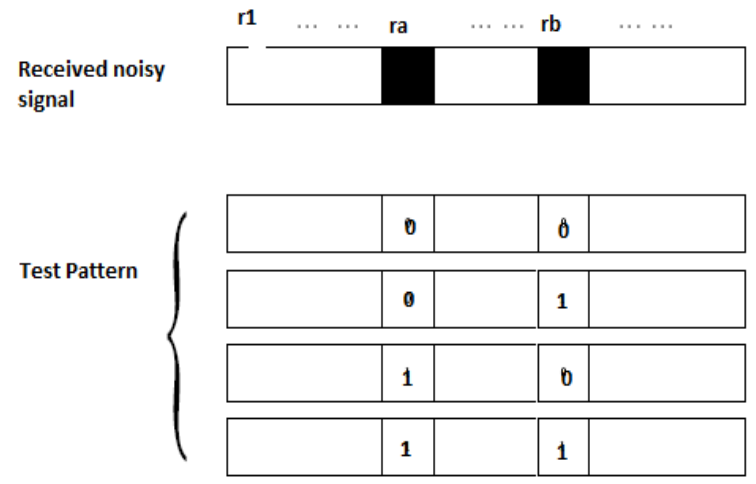

Fig.5. Test Pattern generated by Chase Algorithm, $\mathrm{p}=2$

Steps for Chase Pyndiah Algorithm
1. Generate a reliability sequence rabs $=(|r l|$, $|r 2|, \quad .|\mathrm{rn}+1|)$ and a binary received sequence $\mathrm{y}=$ $(y l, \ldots, y l \ldots, y n)$. Determine the $p$ least reliable bit positions of sequence $\mathbf{y}$ using rabs.

2. Form $2^{p}$ test pattern sequences $\mathrm{t}^{\mathrm{j}}, j=1, \ldots 2^{p}$ which consist of all combinations of binary sequences containing the $p$ least reliable bits of $\mathrm{y}$.

3. Determine the $2^{p}$ perturbed sequences $\mathbf{z} j=\mathbf{y} \oplus \mathfrak{t}^{\mathbf{j}}, j=$ $1,2, \ldots, 2^{p}$.

4. Decode perturbed sequences $z j$ and obtain valid codeword set $\mathrm{c} j$, where $j=1,2, \ldots, 2^{p}$

5. Calculate analog weight of valid codeword set.

6. Estimate the maximum likelihood (ML) codeword d from valid codeword set

7. Compute extrinsic information for received signal and is used to arrive at a solution i.e. the decoded message [4].

The decoding procedure described below is generalized by cascading elementary decoders illustrated in figure 6 . Let us consider the decoding of the rows and columns of a product code $\mathrm{P}$ described above and transmitted on a Gaussian channel. On receiving matrix $[R]$ corresponding to a transmitted codeword $[\mathrm{E}]$, the first decoder performs the soft decoding of the rows (or columns) of P using as input matrix [R]. Soft-input decoding is performed using the Chase algorithm (as given in above Section) and the soft output is computed [7].

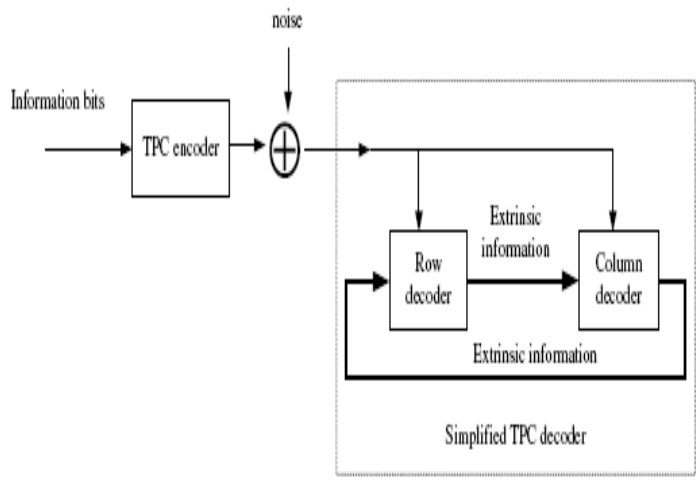

Fig.6. Iterative Decoding of TPC

\section{APPLICATIONS OF TPC}

The iterative decoding of product codes is also known as Block Turbo Code (BTC), because the concept is quite similar to turbo codes based on iterative decoding of concatenated recursive convolutional codes. TPC (eBCH $(64,51,6)$ as constituent code with code rate of 0.635 and 6 iterations in an AWGN channel provides a BER of $10^{-6}$ at an Eb/N0 of $2 \mathrm{db}$ [4].

TPC are the most efficient known codes for high code rate applications. For code rates greater than 0.95, digital transmission systems can transmit data on a Gaussian channel 
at more than $98 \%$ of channel capacity, R/C > 0.98, by guarantee of a minimum distance of 16 or more. While the minimum distance of a CTC can be relatively small. Because of high dmin, typically 16 or higher, there is no error floor for TPC.

Another attractive application for TPC concerns high data rate systems. Indeed, the decoding speed of a TPC can be increased by using several elementary decoders for the parallel decoding of the rows (or columns) of a product code since they are independent [10]. These features, of TPC can be effectively used in a high data rate application like the Wimax 802.16 systems.

\section{SIMULATION RESULT FOR TPC IN 802.16}

In this paper, results of using TPC as the Forward Error Correction (FEC) method in 802.16e systems, is studied. TPC block is generated using eBCH $(64,39)$. Chase Pyndiah algorithm as explained above is applied for 10 iterations for decoding the TPC at the receiver.

TPC is implemented in a 128 point FFT OFDM system. Modulation method used is QPSK. The results, which are plotted using Monte Carlo simulation method for iteration 1, iteration 4, iteration 6 , iteration 8 and iteration 10, are shown in Fig. 7.

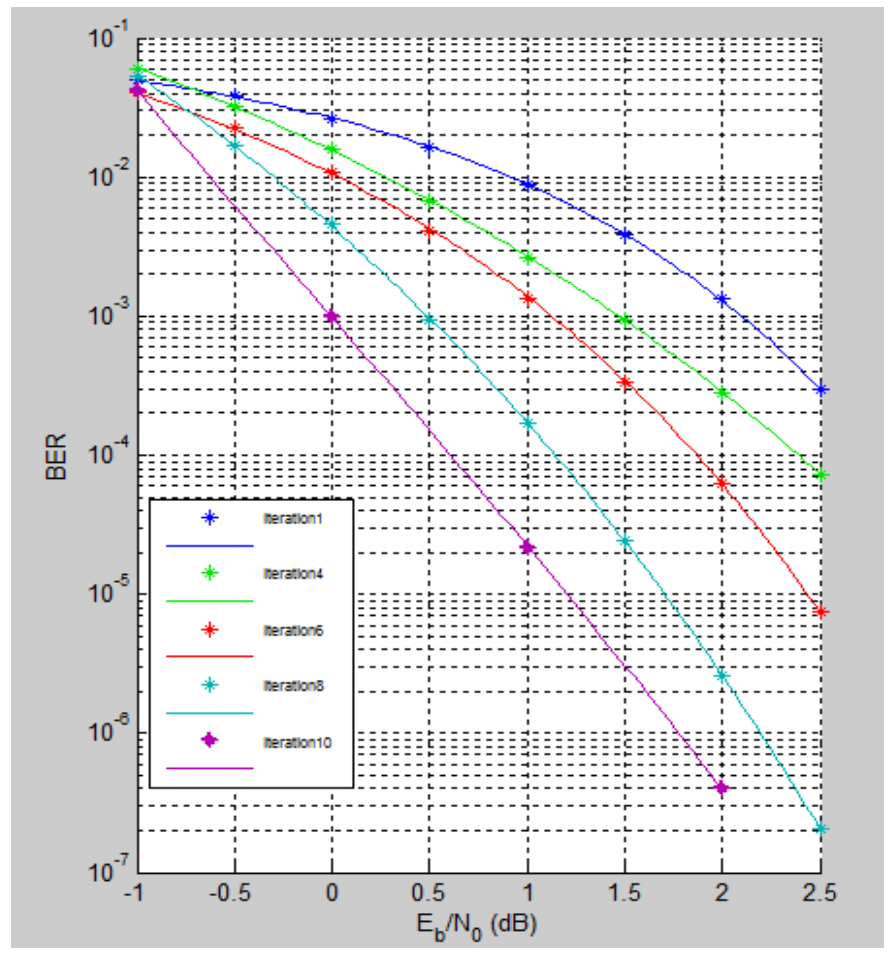

\section{COMPARISON OF PERFORMANCES}

\section{A. COMPARISON OF CTC AND TPC IN 802.16 SYSTEM}

CTC can be used as a FEC method in 802.16e systems. The BER performance of the IEEE 802.16e with CTC decoder using rate $1 / 3$ QPSK code versus the number of iterations is shown in Fig. 8. It is observed that the CTC exhibits a BER greater than $10^{-6}$, but at Eb/N0 ratio of $2.5 \mathrm{db}$ [7].

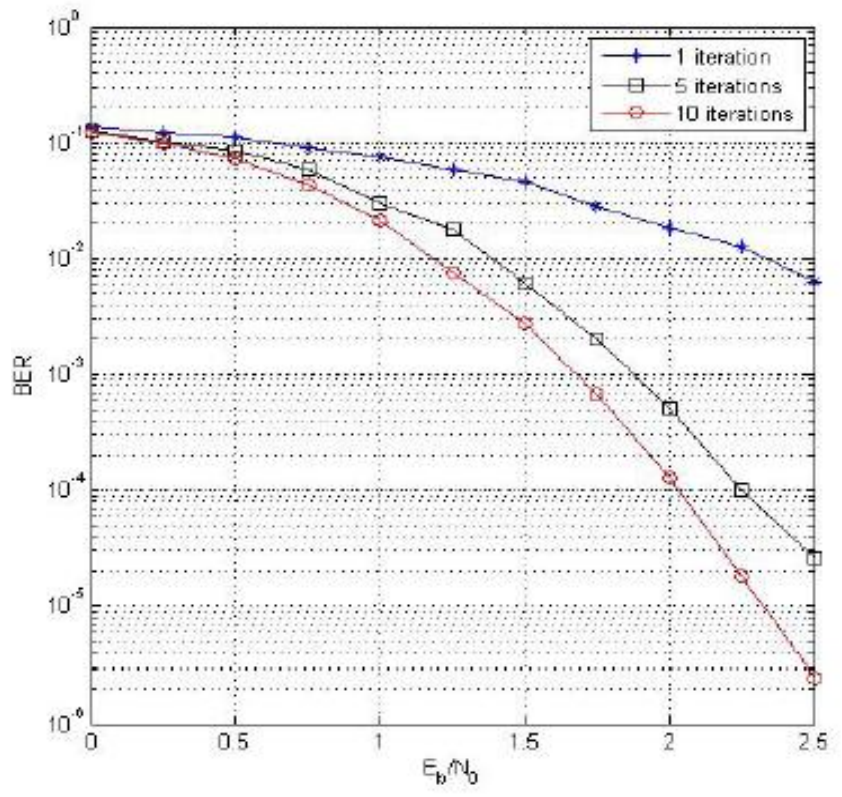

Fig.8. BER verses SNR for CTC in 802.16 systems

Fig. 7, which is plotted for TPC in 802.16e and Fig. 8, which is plotted for CTC in 802.16e, are compared. It is observed that TPC provides the bit error rate less than $10^{-6}$ at $\mathrm{Eb} / \mathrm{N} 0$ of $2.5 \mathrm{db}$, which is better than CTC in 802.16e. From Fig. 7 and 8 the comparative performance of CTC and TPC, also shows that there is a gain of around $1 \mathrm{db}$ in using TPC.

\section{B. COMPARISON OF HYBRID DECODER AND TPC}

Hybrid decoder is a concept, where soft and hard decoding techniques are combined. Initial $\mathrm{m}$ iterations are run by using soft decoding and n-m iterations are run by hard decoding, where $\mathrm{n}$ is the total number of iterations. Value of $\mathrm{n}$ depends on the application and performance [11].

Fig. 9 below shows the BER Vs. Eb/No performance for the standard SISO and Hybrid decoder. Although the number of calculations are reduced, the performance is not very good. Comparing the Fig. 7 and Fig. 9, it is observed that TPC with SISO gives the better performance as compared to hybrid decoder.

Fig.7. BER verses Eb/N0 for TPC in 802.16 systems 


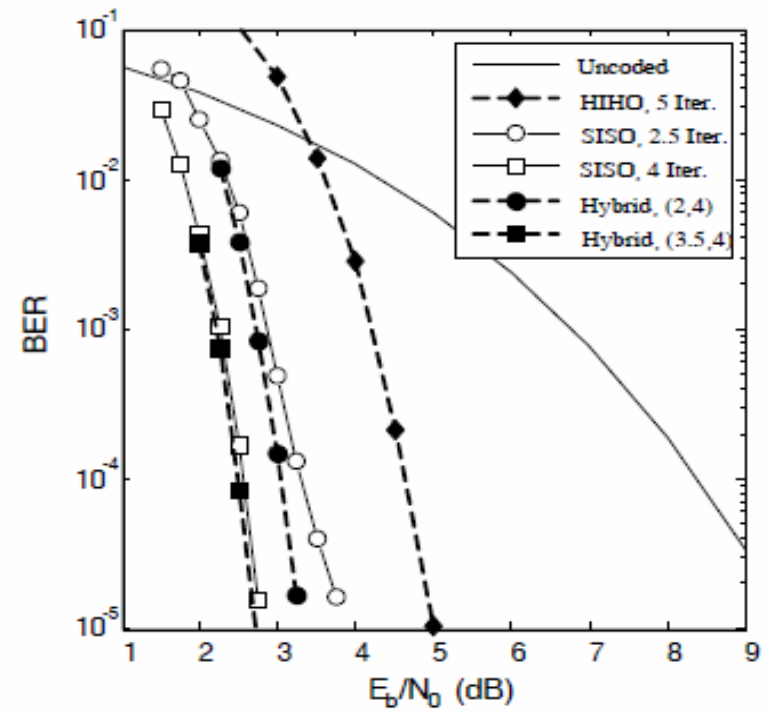

Fig.9. BER verses Eb/No for standard SISO and hybrid decoder for eBCH $(32,21,6)$

\section{COMPLEXITY COMPARISON}

Other advantage of using TPC over CTC in $802.16 \mathrm{e}$ systems is that, there is no need of using the complex interleaver as in CTC. As illustrated in Fig.2 CTC encoder are constructed using interleaver optimization techniques [2]. The need of using complex interleaver and doing its optimization using different techniques for obtaining better BER can be avoided by using TPC. This ultimately reduces the complexity of the decoding technique, which leads to performance improvement.

The table below shows the comparison between CTC and TPC based on the graph which is shown in fig. 7 and fig. 8. It is observed that the performance of TPC is much better as compared to $\mathrm{CTC}$ for a given $\mathrm{Eb} / \mathrm{No}$ range. This can be further improved by increasing the range for $\mathrm{Eb} / \mathrm{No}$ depending on the application.

TABLE I. Eb/N0 verses BER for TPC and CTC

\begin{tabular}{|c|c|c|}
\hline Eb/N0 in db & $\begin{array}{c}\text { BER for TPC at } \\
10^{\text {th }} \text { iteration }\end{array}$ & $\begin{array}{c}\text { BER for TPC at } \\
10^{\text {th }} \text { iteration }\end{array}$ \\
\hline 0 & $1 * 10^{-3}$ & $1 * 10^{-1}$ \\
\hline 0.5 & $1 * 10^{-4}$ & $8^{*} 10^{-2}$ \\
\hline 1 & $1 * 105^{4}$ & $2 * 10^{-2}$ \\
\hline 1.5 & $3^{*} 10^{-6}$ & $3 * 10^{-3}$ \\
\hline 2 & $5^{*} 10^{-7}$ & $1 * 10^{-4}$ \\
\hline 2.5 & - & $\mathbf{3}^{*} \mathbf{1 0}^{-6}$ \\
\hline
\end{tabular}

VIII. CONCLUSIONS

In this paper we had implemented 802.16e system using TPC as forward error correction technique. The results for TPC shows that this can be used for high code rate and data rate applications. TPC with SISO decoding also gives better performance as compared to hybrid decoder. Also there is no need of using complex interleaver or its optimization as in CTC.TPC have satisfied the performance/complexity tradeoff, to meet almost any requirement whether it is a single carrier or multiple carrier OFDM system.

\section{FUTURE SCOPE}

Future scope of this project is improving the performance of TPC by reducing the number of test patterns used to decode the constituent codes of the block of TPC. This is achieved by using syndrome analysis technique. TPC can achieve a throughput of Giga bits per second by parallel decoding of rows and columns. The hardware can be designed to support conflict free interleave memory access model to support parallel decoding [10]. Combining encryption and encoding is the new field of interest for researchers. TPC can be combined with chaotic encryption for different type of data, and performance can be analyzed.

\section{ACKNOWLEDGMENT}

This work was supported by the coordinators and faculty members of Thakur College of Engineering and Technology. Without the support of whom this project would not be a success. We would like to extend our gratitude and sincere thanks to them for their exemplary guidance and encouragement.

\section{REFERNCES}

[1] Paul Guanming Lin "OFDM SIMULATION in MATLAB “ thesis submitted for project in California Polytechnic State University

[2] http://www.aha.com. "Improving Bandwidth Utilization with Turbo Product Codes", pp 32-33

[3] B. Baumgartnery, M. Reinhardtz, G. Richtery, M. Bosserty "Performance of Forward Error Correction for IEEE 802.16e" University of Ulm Germany Dept. of Telecommunications and Applied Information Theory

[4] R. Pyndiah, "Near-Optimum Decoding of Product Codes: Block Turbo Codes" IEEE Trans. Comm., vol. 46, pp.1003-1010

[5] Brian A. Banister, Ph.D "Using Turbo Product Codes in Client Station Uplink for Reduced Power Consumption" Comtech AHA Corporation., pp 1-3

[6] D. Chase "A class of algorithms for decoding block codes with channel measurement information" IEEE Transactions on Information Theory, IT-18 No.1, 1972, pp 172

[7] Yejun He, Francis C.M. Lau *, Chi K. Tse, "Study of bifurcation behavior of two-dimensional turbo product code decoders", pp 503

[8] Mohamed Amr Mokhtar "Study the Performance of Mobile WiMAX Convolutional Turbo Code" Proceedings of the 13th WSEAS International Conference on COMMUNICATIONS, pp 170

[9] Bernard Sklar, Digital Communications Fundamentals and Applications, Pearson, $2^{\mathrm{ND}}$ Edition, 2010, pp 483-489

[10] Richard E. Blahut "Theory and Practice of Error Control Codes" Addison-Wesley, 1983.

[11] Li Zhou, Hengzhu Liu and Botao Zhang "Flexible and high efficiency turbo product code design" National University of Defenece Technology China.

[12] A1-Dweik, S. Le Goff, B. Sharif, IEEE, “ A Hybrid Decoder for Block Turbo Codes", IEEE Transactions on communications, Vol. 57, No. 5, MAY 2009, $1229-1232$. 